\title{
CONTINUOUS ANALOGUE OF ALZER'S INEQUALITY
}

\author{
SU-LING ZHANG, CHAO-PING CHEN AND FENG QI
}

Abstract. Let $b>a>0$ and $\delta>0$ be real numbers, then, for all real $r$,

$$
\frac{b}{b+\delta}<\left(\frac{1}{b-a} \int_{a}^{b} x^{r} d x / \frac{1}{b+\delta-a} \int_{a}^{b+\delta} x^{r} d x\right)^{1 / r}<1 .
$$

Both bounds are best possible.

\section{Introduction}

It has been shown in $[3,12]$ that

$$
\frac{n}{n+1}<\left(\frac{1}{n} \sum_{i=1}^{n} i^{r} / \frac{1}{n+1} \sum_{i=1}^{n+1} i^{r}\right)^{1 / r}<1
$$

for all natural numbers $n$, and all real $r$. Both bounds of (1) are best possible. This extends a result given by H. Alzer [1], who established this inequality for $r>0$. For $r>0$, several easy proofs of (1) have been published by different authors, see $[2,11,15]$. For convience, we call (1) Alzer's inequality.

In this paper, we present a continuous analogue of (1) as follows:

Theorem. Let $b>a>0$ and $\delta>0$ be real numbers, then, for all real $r$,

$$
\frac{b}{b+\delta}<\left(\frac{1}{b-a} \int_{a}^{b} x^{r} d x / \frac{1}{b+\delta-a} \int_{a}^{b+\delta} x^{r} d x\right)^{1 / r}<1 .
$$

Both bounds are best possible.

Remark. Our theorem extends a result given by F. Qi [10], who established the inequality (2) for $r>0$. In fact, (2) can be written as

$$
\frac{b}{b+\delta}<\frac{L_{r}(a, b)}{L_{r}(a, b+\delta)}<1
$$

Received and revised June 24, 2004.

2000 Mathematics Subject Classification. 26D15.

Key words and phrases. Alzer's inequality, continuous analogue, generalized logarithmic mean. The authors were supported in part by NNSF (\#10001016) of China, SF for the Prominent Youth of Henan Province (\#0112000200), SF of Henan Innovation Talents at Universities, Doctor Fund of Jiaozuo Institute of Technology, China 
where $L_{r}(a, b)$ denotes the generalized logarithmic mean of two positive numbers $a, b$. For convience, let us recall that the generalized logarithmic mean $L_{r}(a, b)$ of two positive numbers $a, b$ is defined in $[5,13,14]$ for $a=b$ by $L_{r}(a, b)=a$ and for $a \neq b$ by

$$
\begin{aligned}
L_{r}(a, b) & =\left(\frac{b^{r+1}-a^{r+1}}{(r+1)(b-a)}\right)^{1 / r}, \quad r \neq-1,0 ; \\
L_{-1}(a, b) & =\frac{b-a}{\ln b-\ln a}=L(a, b) ; \\
L_{0}(a, b) & =\frac{1}{e}\left(\frac{b^{b}}{a^{a}}\right)^{1 /(b-a)}=I(a, b) .
\end{aligned}
$$

$L(a, b)$ and $I(a, b)$ are respectively called the logarithmic mean and exponential mean of two positive numbers $a, b$. When $a \neq b, L_{r}(a, b)$ is a strictly increasing function of $r$. In particular,

$$
\lim _{r \rightarrow-\infty} L_{r}(a, b)=\min \{a, b\}, \quad \lim _{r \rightarrow+\infty} L_{r}(a, b)=\max \{a, b\} .
$$

\section{Proof of (3)}

For $r=-1$. Then the left hand inequality of (3) is

$$
\frac{b}{b+\delta}<\frac{L(a, b)}{L(a, b+\delta)}
$$

which is equivalent to

$$
\begin{gathered}
\frac{b+\delta-a}{(b+\delta)[\ln (b+\delta)-\ln a]}<\frac{b-a}{b(\ln b-\ln a)}, \\
\frac{[a /(b+\delta)]-1}{\ln [a /(b+\delta)]}<\frac{(a / b)-1}{\ln (a / b)} .
\end{gathered}
$$

Define the function $f$ by

$$
f(t)=\frac{t-1}{\ln t} \quad(0<t<1)
$$

Differentiation yields

$$
\begin{aligned}
f^{\prime}(t) & =\frac{1-t+t \ln t}{t(\ln t)^{2}}=\frac{1}{t(\ln t)^{2}}\left(1-t-t \sum_{n=1}^{\infty} \frac{(1-t)^{n}}{n}\right) \\
& >\frac{1}{t(\ln t)^{2}}\left(1-t-t \sum_{n=1}^{\infty}(1-t)^{n}\right)=0
\end{aligned}
$$

which implies (4). 
For $r=0$. Then the left hand inequality of $(3)$ is

$$
\frac{b}{b+\delta}<\frac{I(a, b)}{I(a, b+\delta)}
$$

which is equivalent to

$$
\begin{aligned}
\frac{1}{b+\delta}\left(\frac{(b+\delta)^{b+\delta}}{a^{a}}\right)^{1 /(b+\delta-a)} & <\frac{1}{b}\left(\frac{b^{b}}{a^{a}}\right)^{1 /(b-a)}, \\
\left(\frac{b+\delta}{a}\right)^{1 /(b+\delta-a)} & <\left(\frac{b}{a}\right)^{1 /(b-a)}, \\
L(a, b)=\frac{b-a}{\ln b-\ln a} & <\frac{b+\delta-a}{\ln (b+\delta)-\ln a}=L(a, b+\delta) .
\end{aligned}
$$

Since the logarithmic mean $L(a, b)$ is strictly increasing with respect to the two variables $a$ and $b,(5)$ holds obviously.

For $r(r+1) \neq 0$. Then the left hand inequality of $(3)$ is equivalent to

$$
\frac{b^{r+1}-a^{r+1}}{b-a} / \frac{(b+\delta)^{r+1}-a^{r+1}}{b+\delta-a} \gtrless \frac{b^{r}}{(b+\delta)^{r}}, \text { according as } r \gtrless 0 \text {. }
$$

i.e. (since $(b+\delta)^{r+1}-b^{r+1} \gtrless 0$ according as $r+1 \gtrless 0$ ),

$$
\frac{b^{r+1}-a^{r+1}}{(b-a) b^{r}} \gtrless \frac{(b+\delta)^{r+1}-a^{r+1}}{(b+\delta-a)(b+\delta)^{r}}, \text { according as } r(r+1) \gtrless 0 \text {, }
$$

i.e.

$$
\frac{1-(a / b)^{r+1}}{1-(a / b)} \gtrless \frac{1-[a /(b+\delta)]^{r+1}}{1-[a /(b+\delta)]}, \text { according as } r(r+1) \gtrless 0 \text {. }
$$

By the mean value theorem for derivatives,

$$
\begin{aligned}
\frac{\mathrm{d}}{\mathrm{d} x}\left(\frac{1-x^{r+1}}{1-x}\right) & =\frac{1}{1-x}\left[\frac{1-x^{r+1}}{1-x}-(r+1) x^{r}\right] \\
& =\frac{(r+1)\left(\xi^{r}-x^{r}\right)}{1-x} \gtrless 0 \quad(0<x<\xi<1),
\end{aligned}
$$

according as $r(r+1) \gtrless 0$, which implies $(6)$.

Since the generalized logarithmic mean $L_{r}(a, b)$ is strictly increasing with respect to the two variables $a$ and $b$ (see $[4,6,7,8,9]$ ), the right hand inequality of $(3)$ holds obviously.

It is clear that

$$
\lim _{r \rightarrow+\infty} \frac{L_{r}(a, b)}{L_{r}(a, b+\delta)}=\frac{b}{b+\delta}, \quad \lim _{r \rightarrow-\infty} \frac{L_{r}(a, b)}{L_{r}(a, b+\delta)}=1 .
$$

Thus, the both bounds given in (3) are best possible. The proof of (3) is complete. 


\section{References}

[1] H. Alzer, On an inequality of H. Minc and L. Sathre, J. Math. Anal. Appl. 179 (1993), 396-402.

[2] Ch.-P. Chen and F. Qi, Notes on proofs of Alzer's inequality, O. Math. Mag. 11 (2003), 29-33.

[3] Ch.-P. Chen and F. Qi, The inequality of Alzer for negative powers, O. Math. Mag. 11 (2003), 442-445.

[4] Ch.-P. Chen and F. Qi, A new proof for monotonicity of the generalized weighted mean values, Adv. Stud. Contemp. Math. (Kyungshang) 5 (2003), 13-16.

[5] L. Galvani, Dei limiti a cui tendono alcune media, Boll. Un. Mat. Ital. 6 (1927), 173-179.

[6] E. B. Leach and M. C. Sholander, Extended mean values, Amer. Math. Monthly 85 (1978), 84-90.

[7] E. B. Leach and M. C. Sholander, Extended mean values II, J. Math. Anal. Appl. 92 (1983), 207-223.

[8] F. Qi and Q.-M. Luo, A simple proof of monotonicity for extended mean values, J. Math. Anal. Appl. 224 (1998), 356-359.

[9] F. Qi, S.-L. Xu, and L. Debnath, A new proof of monotonicity for extended mean values, Internat. J. Math. Math. Sci. 22 (1999), 417-421.

[10] F. Qi, An algebraic inequality, J. Inequal. Pure Appl. Math. 2 (2001), Art. 13.

[11] J. Sándor, On an inequality of Alze, J. Math. Anal. Appl. 192 (1995), 1034-1035.

[12] J. Sándor, On an inequality of Alzer, II, O. Math. Mag. 11 (2003), 554-555.

[13] K. B. Stolarsky, Generalizations of the logarithmic mean, Math. Mag. 48 (1975), 87-92.

[14] K. B. Stolarsky, The power and generalized logarithmic means, Amer. Math. Monthly 87 (1980), 545-548.

[15] J. S. Ume, An elementary proof of H. Alzer's inequality, Math. Japon. 44 (1996), 521-522.

Department of Basic Courses, Jiaozuo University, Jiaozuo City, Henan 454003, China.

Department of Applied Mathematics and Informatics, Research Institute of Applied Mathematics, Henan Polytechnic University, \#142, Mid-Jiefang Road, Jiaozuo City, Henan 454000, China.

E-mail: chenchaoping@sohu.com; chenchaoping@hpu.edu.cn

Department of Applied Mathematics and Informatics, Research Institute of Applied Mathematics, Henan Polytechnic University, \#142, Mid-Jiefang Road, Jiaozuo City, Henan 454000, China.

E-mail: qifeng@hpu.edu.cn 